AperTO - Archivio Istituzionale Open Access dell'Università di Torino

\title{
Automatic GTV contouring applying anomaly detection algorithm on dynamic FDG PET images
}

\section{This is the author's manuscript}

Original Citation:

\section{Availability:}

This version is available http://hdl.handle.net/2318/1558174

since 2017-05-12T15:35:45Z

Publisher:

Elvesier

Published version:

DOI:10.1016/j.ejmp.2016.01.343

Terms of use:

Open Access

Anyone can freely access the full text of works made available as "Open Access". Works made available under a Creative Commons license can be used according to the terms and conditions of said license. Use of all other works requires consent of the right holder (author or publisher) if not exempted from copyright protection by the applicable law. 


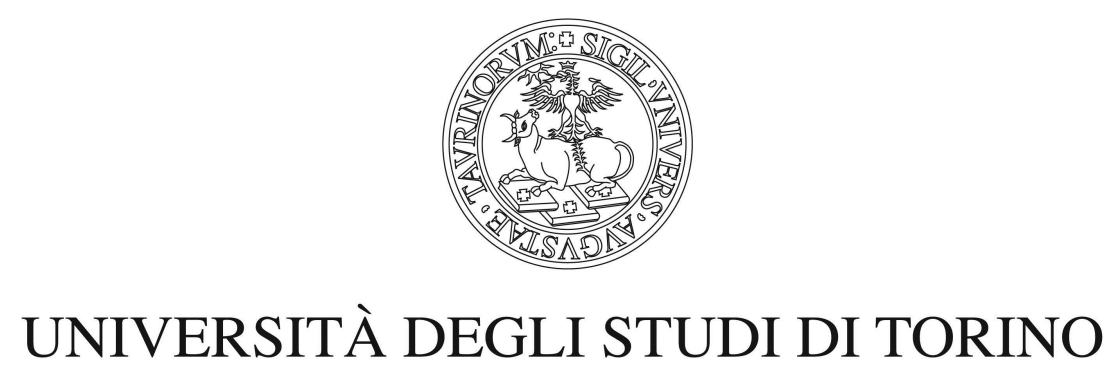

This is an author version of the contribution published on:

C. Bracco, F. Verdoja, M. Grangetto, A. Di Dia, M. Racca, T. Varetto, and M. Stasi. Automatic GTV contouring applying anomaly detection algorithm on dynamic FDG PET images. Physica Medica, vol. 32, no. 1, p. 99, Feb. 2016. 10.1007978-3-319-23231-7_33.

The definitive version is available at: 


\begin{abstract}
Introduction The aim of this work is to show the results of GTV automatic segmentation based on dynamic PET acquisition. With respect to single voxel segmentation the temporal information is used to improve quality of GTV delineation. The segmentation algorithm proposed exploits the theoretic assumption that FDG uptake over time in cancer cells is very different from the one in normal tissues and therefore in this study anomaly detection is used to look for tumor peculiar-anomalous TACs.
\end{abstract}

Material and Methods For each patient two list mode datasets of images were acquired. The first one scan (basal) was acquired one hour after FDG injection and reconstructed as static frame. The last one (delayed) was acquired half one hour after the first scan and reconstructed as dynamic scan. Two delayed scans were registered to the basal scan. A modified version of the RX Detector was used. RX Detector usually works in RGB, but in this study its use on TACs has been explored passing the three grayscale images in place of the three channels of RGB. The resulting single image, which actually is a matrix of Mahalanobis distances, presents values that are very high for voxels whose TAC has anomalous temporal behavior. Finally, threshold segmentation is performed on the distance matrix. On a dataset of 10 patients segmentation techniques present in the literature working on single PET scan have been implemented as well as segmentation techniques based on RX Detector output.

Results Spatial overlap index (SOI) was used as metric to evaluate the segmentation accuracy. All of the segmentation algorithms implemented on RXD output show better SOI $(0.507 \pm 0.158)$ than algorithm based on SUV, i.e. Brambilla, SOI $0.278 \pm 0.236$. A manual contour drawn by experienced Nuclear Physician was the reference.

Conclusion Although a small dataset, the segmentation of dynamic PET images based on RXD output seems to be promising. 